\title{
STho Maintenance Azacitidine after Hematopoietic Stem Cell Transplantation for Relapse \\ Prevention in Acute Myeloid Leukemia and Myelodisplastic Syndrome
}

André Dias Américo, Larissa Lanes Teixeira, Mariana Nassif Kerbauy, Cinthya Correa da Silva, Eduardo Cerello Chapchap,

Fabio Pires de Souza, Andreza Alice Feitosa Ribeiro, Nelson Hamerschlak

\section{Introduction}

Relapse after HSCT remains one of the main causes of transplant mortality in AML and MDS. Azacitidine has been reported to improve graftversus-leukemia, but clinical data on phase I/lI trials have shown conflicting results.

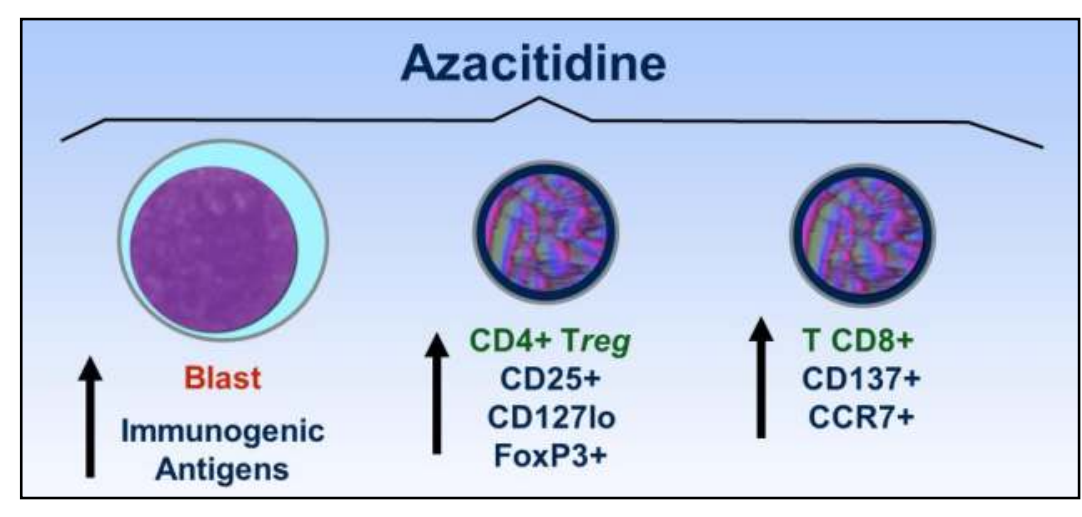

Figure 1. Azacitidine effects post HSCT

\section{Objectives}

To assess the effect of maintenance azacitidine following HSCT on clinical outcomes.

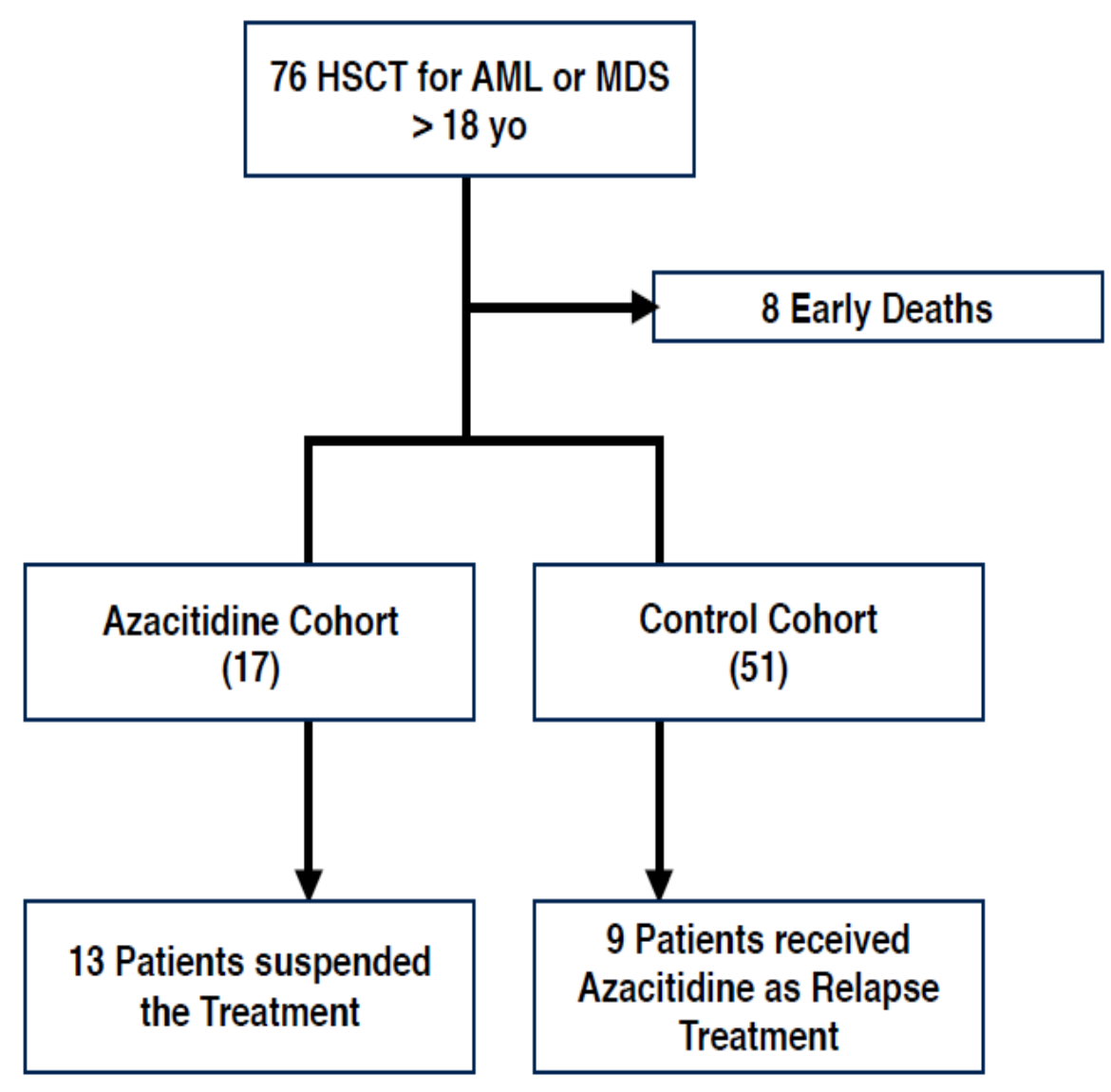

Figure 2. Flow Chart
Table 1. Baseline clinical characteristics

\begin{tabular}{|c|c|c|c|}
\hline Variable & Azacitidine & Control & $p$ \\
\hline Age (mean) & 51,65 & 56,02 & 0,2 \\
\hline Male sex & $52,9 \%$ & 56,9 & 1 \\
\hline $\begin{array}{l}\text { MDS } \\
\text { Diagnosis }\end{array}$ & $17,6 \%$ & 39,2 & 0,18 \\
\hline $\begin{array}{l}\text { High Risk } \\
\text { Kariotype }\end{array}$ & $41,2 \%$ & $31,4 \%$ & 0,65 \\
\hline $\begin{array}{l}\text { DRI } \\
\text { Intermediat } \\
\text { e }\end{array}$ & $47,1 \%$ & $58,5 \%$ & 0,69 \\
\hline $\begin{array}{l}\text { Transplant } \\
\text { Type }\end{array}$ & & & 0,31 \\
\hline MSD & $23,5 \%$ & $39,2 \%$ & \\
\hline MUD & $35,3 \%$ & $37,3 \%$ & \\
\hline Haplo & $41,2 \%$ & $23,5 \%$ & \\
\hline MAC & $64,7 \%$ & $43,1 \%$ & 0,1 \\
\hline $\begin{array}{l}\text { PBSC Graft } \\
\text { Source }\end{array}$ & $41,2 \%$ & $35,3 \%$ & 0,67 \\
\hline $\begin{array}{l}\text { Azacitidine } \\
\text { use }\end{array}$ & $100 \%$ & $21,6 \%$ & $\begin{array}{l}0,00 \\
1\end{array}$ \\
\hline $\begin{array}{l}\text { Azacitidine } \\
\text { cycles } \\
\text { (median) }\end{array}$ & 8 & 3,82 & $\begin{array}{l}0,20 \\
5\end{array}$ \\
\hline $\begin{array}{l}\text { Karnosfsky } \\
>90 \%\end{array}$ & $82,4 \%$ & $78,4 \%$ & 1 \\
\hline
\end{tabular}

$>90 \%$

Materials and Methods
We conducted a retrospective analysis of medical
charts from patients aged 18 years or older,
diagnosed with either MDS or AML who received
bone marrow allograft. Retrieved data regarding
their baseline characteristics (age, year of
transplantation, etc.), disease biology (karyotype)
HSCT (conditioning, use of azacitidine as
maintenance, disease relate index, etc.) and
outcomes (overall survival, relapse rates and
relapse free survival).

\section{Results}

Seventy-Six patients received an allograft as treatment for MDS or AML at our institution from 2011 to 2018 (8 patients were excluded due to precocious deaths - in order to avoid a survival bias). Seventeen patients received azacitidine as maintenance (i.e. prior to relapse) while the remaining 51 patients composed the control group ( 9 of these received azacitidine, but only after relapse - see Figure 2). The 2 year OS rates for the aza and control groups were $67,1 \%$ and $37,1 \%$ but this difference was not statistically significant through log-rank analysis $(p=0,12)$ The PFS rates at 2 years were $56,6 \%$ and $39,1 \%$ for aza and control groups, while the cumulative incidence of relapse was $38,6 \%$ and $28,4 \%$, but these differences were not statistically different $(\mathrm{p}=0,22$ and 0,53$)$. We then decided to conduct a Cox proportional hazards to assess independent azacitidine effect, controlling for disease related index (see Table 2). This analysis showed marginally independent protective effect for aza on OS and PFS with HR 0,41 (0,17-1,0, $\mathrm{p}=0,009)$ and with $\operatorname{HR} 0,49(0,22-1,09, p=0,005)$.

Table 2. Cox Proportional Hazard

\begin{tabular}{lllll}
\hline OS & $\begin{array}{l}\text { Univariate } \\
\text { Analysis } \\
\text { HR (95\% } \\
\text { Cl) }\end{array}$ & $\begin{array}{l}\text { Multivariat } \\
\mathbf{e} \\
\text { Analysis } \\
\text { HR (95\% } \\
\text { Cl) }\end{array}$ & $\mathbf{p}$ \\
\hline Aza & $\begin{array}{l}0,5(0,2- \\
1,21)\end{array}$ & 0,1 & $\begin{array}{l}0,41(0,17- \\
1,0)\end{array}$ & 0,009 \\
DRI & $0,44(0,22-$ & & $0,39(0,19-$ & \\
Interm & $0,89)$ & & $0,78)$ & \\
\hline PFS & & & & \\
\hline Aza & $0,61(0,28-$ & 0,2 & $0,49(0,22-$ & 0,005 \\
& $1,34)$ & & $1,09)$ & \\
DRI & $0,41(0,21-$ & 0,008 & $0,36(0,38-$ & \\
Interm & $0,79)$ & & $0,71)$ \\
ediate & & & & \\
\hline
\end{tabular}
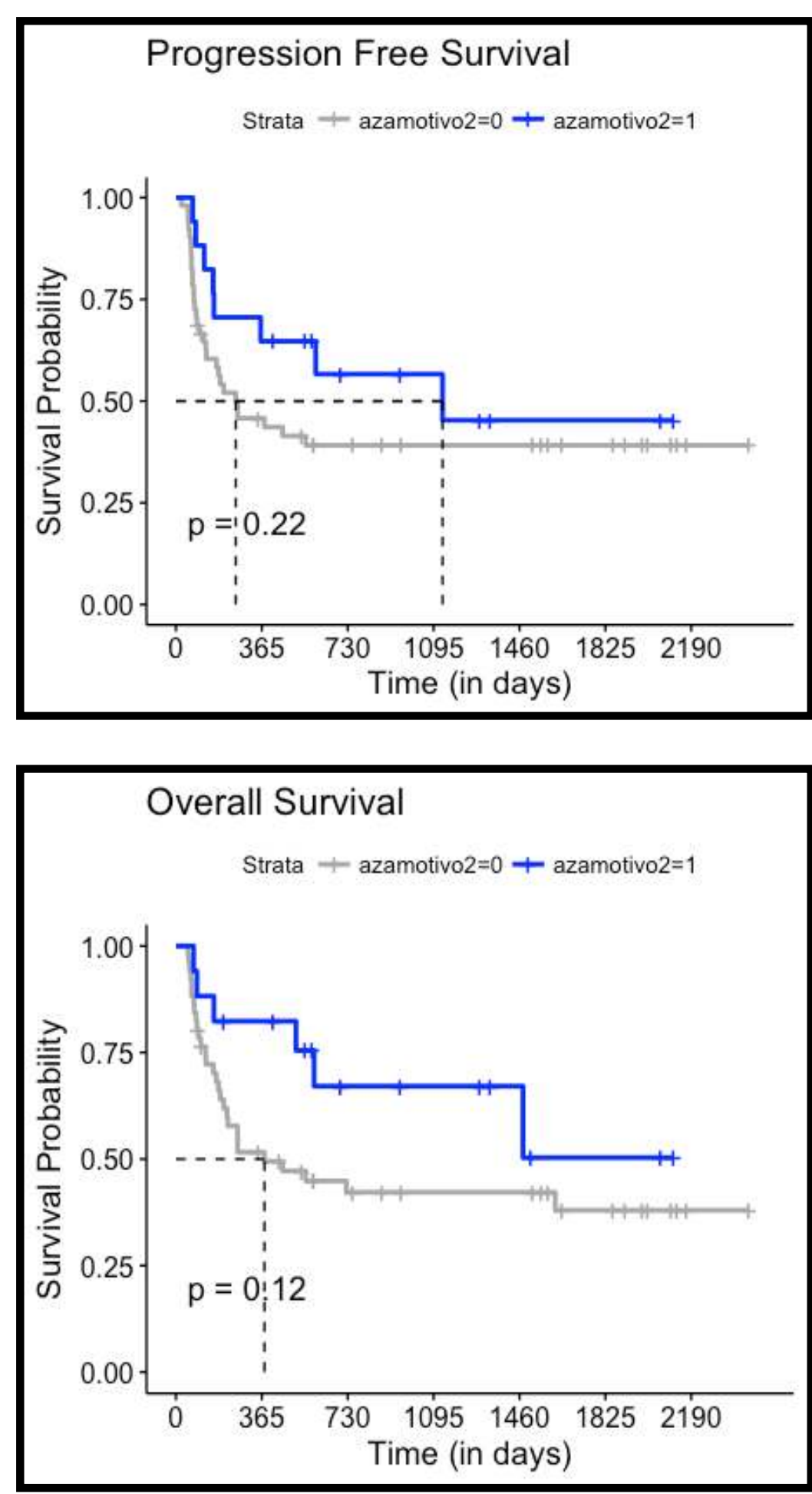

Figure 3. PFS and OS KM Curves

\section{Discussion}

Our data showed marginally positive results and a tendency in favor of the azacitidine group, the use of hypomethilating agents as relapse prophylaxis should be evaluated in the setting of a controlled clinical trial

References
1. de Lima, M et al, Cancer 2010;116:5420-31
2. Goodyear, OC et al, Blood
2012;119(14):3361-69
3. Maples, KT et al, Leuk \& Lymph 2018;

